\title{
LA SUBORDINAZIONE FEMMINILE NEL ROMANZO LIBERTINO FRANCESE SETTECENTESCO E NELLE MÉMOIRES D'UNE HONNÊTE FEMME DI FRANÇOIS- ANTOINE CHEVRIER
}

\author{
Luisa MESSINA \\ Università di Palermo
}

\begin{abstract}
Résumé (Fr) : En 1753 Chevrier publie le roman Mémoires d'une honnête femme écrits par ellemême où l'écrivain vise à subvertir le genre des mémoires en donnant voix à une femme, à la différence des mémoires traditionnels relatant une vie entière d'un homme, en focalisant l'attention sur une période brève comprenant le mariage de Julie et son choix de finir ses jours au couvent. En utilisant les éléments typiques du roman libertin et du roman sentimental, Chevrier y unit les composantes du roman noir et du roman picaresque en passant d'un genre à l'autre sans transition. En considérant l'hétérogénéité des références intertextuelles, il faut constater que Mémoires d'une honnête femme a particulièrement subi de différentes influences littéraires pour remettre en question les règles imposées par la haute société mondaine.
\end{abstract}

Mots-clés (Fr) : Chevrier ; roman ; libertin ; dix-huitième siècle ; femme ; subordination.

\begin{abstract}
En): In 1753 Chevrier publishes Mémoires d'une honnête femme écrits par elle-même where the author aims to subvert conventional mémoires giving voice to a female character, in contrast to traditional mémoires describing a whole life of a male character. Chevrier focuses on a short period comprising Julie's marriage and her choice to finish her days in a convent. Using typical elements characterizing libertine and sentimental novels, the writer joins some components defining roman noir (Noir fiction) and picaresque novel passing from one genre to another without any sense of transition. Taking into consideration heterogeneous inter-textual references, we dare to claim that Mémoires d'une honnête femme has been subjected to different literary influences with the objective of questioning some rules which were imposed by the high society of the period concerned.
\end{abstract}

Keywords (En): Chevrier; novel; libertine; eighteenth century; woman; submission.

\section{Il ruolo femminile nei romanzi libertini francesi del Settecento}

I romanzi libertini francesi del Settecento si incentrano tradizionalmente sulla nascita di un intrigo amoroso al di fuori del matrimonio mostrando, fra le altre cose, un'evidente diseguaglianza tra uomo e donna in termini di rapporti di potere. Una fanciulla a cui viene comunicato l'imminente matrimonio combinato non possiede alcun potere decisionale: l'adolescente settecentesca è, quindi, costretta ad accettare questa imposizione, condizione certamente preferibile ad un eventuale ritorno in convento, perché il nuovo status sociale derivante dalle nozze le apre un nuovo mondo in virtù della posizione del marito (DE GONCOURT, 1862:130). A ciò si aggiunge che la sessualità ai tempi dell'Ancien Régime implica sicuramente una netta separazione tra i doveri coniugali di alcova, per i quali la moderazione è considerata una delle condizioni imprescindibili al fine di generare una prole numerosa, e la sessualità extraconiugale, i cui smoderati eccessi sono al contrario considerati poco fecondi (MATTHEWS-GRIECO, 2005:185). Tale contesto storicosociale implica pertanto da una parte una considerevole frattura tra il matrimonio e il piacere sessuale e, dall'altra, una maggiore consapevolezza da parte delle donne (mogli adultere e figlie ribelli) nel voler rivendicare i propri diritti sessuali 
constatando che i tradimenti commessi dagli uomini vengono incondizionatamente approvati e tollerati dalla società.

La maggior parte dei romanzi libertini del periodo in questione oscilla tra la rappresentazione dell'utopia di un'illimitata apoteosi dei sensi e quella della trasgressione dei tabù. L'utopia galante, che presuppone il trionfo dei valori femminili e l'instaurazione di rapporti rilassati tra i sessi, si rivela fragile in quanto il predominio maschile continua a essere una realtà tangibile nella società d'Ancien Régime. Buona parte dei romanzi galanti dell'epoca classica della letteratura francese, e non solamente quelli libertini, mostrano come i principi della galanteria finiscano per essere sovvertiti: la presunta uguaglianza dei sessi è sostituita da un sistema in cui la donna, sedotta o intimidita, si ritrova spesso nella condizione di vittima (HÖLZLE, 2012:11). Le donne, dunque, sono costrette non solo a tollerare i tradimenti del marito, ma a nascondere le loro eventuali relazioni adultere per paura di essere perdute.

Ciò nonostante, la presenza femminile è una costante nella letteratura libertina, all'interno della quale un personaggio può assumere variabilmente il ruolo di cacciatrice o quello di preda. Va inoltre precisato che nel Settecento la donna appartenente all' aristocrazia o all'alta borghesia acquisisce una funzione sempre più determinante in virtù del ruolo assegnatole dall'uomo, poiché nel periodo compreso tra il 1720 e il 1789 la funzione sociale femminile viene rimpiazzata da quella sessuale. Sebbene la donna sia tradizionalmente considerata un semplice oggetto del desiderio maschile, alcuni personaggi descritti nei romanzi libertini (Cidalise ne La nuit et le moment di Crébillon; la marchesa Merteuil ne Les liaisons dangereuses; Félicia nell'omonimo romanzo di Nerciat) hanno certamente provato a opporsi a questa umiliante condizione di subordinazione sociale e sessuale. Il perseguimento di una condotta libertina però è un' esperienza estremamente pericolosa per le donne o, comunque, destinata a fallire: a differenza del libertinaggio liberamente ostentato dagli uomini, quello femminile è condannato socialmente in quanto più solitario, audace e segreto rispetto a quello maschile. Il libertinaggio femminile è, quindi, giudicato come insidioso e fonte di disgrazie o tutt'al più viene a malapena tollerato se il suo fine è l'iniziazione amorosa di un giovane nobile (JATON, 1982: 155).

Considerando l'importanza della reputazione femminile, la donna agisce mascherandosi e ricorrendo alle armi della finzione e dell' astuzia, riuscendo persino a soggiogare altre figure femminili. Tuttavia, se si considera ad esempio il caso della marchesa de Merteuil, la sua maschera è destinata in ogni caso alla distruzione: in seguito alla morte di Valmont, viene esclusa dai salotti parigini, per lei una morte sociale ignobile che equivale alla morte per spada toccata allo stesso Valmont. Il tragico epilogo della marchesa manifesta il fallimento del suo vano tentativo di ribaltare lo stato delle cose relativo alla sottomissione femminile, ma anche quello legato al principio stesso del libertinaggio.

Altre donne emergenti dalla produzione libertina cercano di condurre un'esistenza virtuosa rischiando però di diventare facili vittime di un libertino navigato a cui la nobildonna ritiene di poter resistere. Credendo di poter accettare la fiducia e l'amicizia offerte, la devota inizia una fitta corrispondenza con lo spasimante libertino: basti pensare alle eroine di Crébillon (la marchesa de $\mathbf{M}^{* * *}$ ), di Dorat (Madame de Syrcé) e di Laclos (Mme de Tourvel). Diversamente da queste 
donne virtuose minacciate dal libertinaggio, la protagonista del romanzo di Chevrier Mémoires d'une honnête femme écrits par elle-même fornisce un raro esempio di donna dalla forte tempra morale in grado di resistere alle insidie del desiderio.

\section{Il ruolo femminile nel romanzo Mémoires d'une honnête femme écrits par elle-même di François-Antoine Chevrier}

François-Antoine Chevrier è conosciuto come uno degli scrittori francesi settecenteschi tra i più odiati e disprezzati del suo tempo a causa della sua vena satirica e polemica che lo costrinse a vivere lontano dalla Francia. La vera intenzione di Chevrier era, a ben vedere, quella di criticare i costumi delle classi sociali più abbienti, ultimi rappresentanti di una società decadente, e i comportamenti di un governo corrotto. Se si considera la presenza femminile all'interno della società d'Ancien Régime descritta da Chevrier, è possibile constatare che la donna svolge un ruolo fondamentale nella produzione e nella vita dello scrittore, il quale sebbene manifesti uno spiccato gusto per il gentil sesso esprime nei suoi confronti una critica acerba che non verrà mai meno. In realtà Chevrier critica solo alcune tipologie femminili: da una parte, si scaglia contro le attrici e le cantanti che vendono il loro talento e, dall' altra, contro le nobildonne lascive che adottano una condotta amorale e volitiva simile a quella ostentata dal sesso maschile. Lo scrittore lorenese, talvolta, mostra l'antitesi della depravazione femminile concentrandosi su alcune figure particolarmente virtuose come Julie, protagonista delle Mémoires d'une honnête femme écrits par elle-même, romanzo pubblicato nel 1753. Questa opera racconta un breve periodo della vita della protagonista Julie, ossia il periodo compreso tra il suo matrimonio e la clausura volontaria in convento. Julie è una giovane donna totalmente in balia delle decisioni coercitive imposte dagli uomini della sua vita, inizialmente dal padre e successivamente dal marito: seguendo gli ordini imposti dalla famiglia, l'adolescente Julie deve lasciare il convento in cui si trova al fine di sposare il conte de Courmont, sebbene sia innamorata del cavaliere de Nalbour. A seguito della decisione del marito di trasferirsi a Parigi, Julie fronteggia numerose situazioni spiacevoli, come la gelosia furiosa del marito, che l'accusa ingiustamente facendola momentaneamente rinchiudere in un convento destinato alle adultere. Sebbene il marito la tradisca ripetutamente, Julie è risoluta a preservare la propria condotta virtuosa rispettando persino la promessa fatta al coniuge morente di rimanere vedova per il resto della sua vita. Turbata dalla morte del suo unico figlio, Julie decide di passare il resto dei suoi giorni nel convento delle Orsoline, dove scrive le sue memorie.

Il titolo completo del romanzo Mémoires d'une honnête femme, écrites par ellemême, et publiées par M. Chevrier contiene un chiaro riferimento al romanzo Mémoires d'un honnête homme di Prévost (1728). Mentre Prévost ha mostrato i dissidi interiori di un eroe scisso tra il perseguimento della morale e la ricerca del piacere, Chevrier ha invece individuato una rottura significativa tra i sentimenti dell'eroina e un io sociale totalmente differente. Scegliendo quindi di dar voce a un personaggio femminile, Chevrier ha deciso di focalizzare l'attenzione sulla problematicità della psicologia e del desiderio femminile nella misura in cui la moralità della donna settecentesca è severamente giudicata in termini di verginità, 
castità e fedeltà (BOKOBZA KAHAN, 2005: 16-17). La scrittura sotto forma di memoriale rimanda certamente ad altri romanzi libertini incentrati su figure femminili: ne La Tourière des carmélites (1745) di Meusnier de Querlon la protagonista Agnès, meglio conosciuta come Sainte Nitouche, racconta le sue memorie sotto forma di lettere indirizzate alla sorella Geneviève, superiore della maison de force a Salpêtrière, ripercorrendo le tappe della sua giovinezza voluttuosa. L'ambizione a condurre una vita virtuosa è certamente paragonabile a quella di altre eroine di romanzi dello stesso tipo come Madame de Syrcé (Les malheurs de l'inconstance di Dorat) e Madame de Tourvel (Les liaisons dangeureuses di Laclos), che soccombono a causa della loro condotta libertina, fonte di laceranti sensi di colpa. Considerando il topos della donna virtuosa nel quadro della letteratura europea settecentesca, l'integrità morale di Julie potrebbe essere certamente paragonata a quella delle eroine inglesi Pamela (1740) e Clarissa (1747-1748) di Richardson, autore che meglio di ogni altro ha fissato il modello per questa tipologia di personaggio letterario. La strenua lotta di Julie per preservare la sua virtù, inoltre, permette di ritrovare un altro elemento comune a un altro romanzo di Duclos, ossia L'histoire de Madame de Luz (1741), dove la virtù della protagonista è minata da ripetute violenze subite diversamente da Julie, che si limita a lamentarsi dei costumi corrotti dell'epoca. L'histoire de Madame de Luz di Duclos, in cui l'eroina è perseguitata e oltraggiata fino alla sua morte, decreta il fallimento di Saint-Géran, l'uomo che avrebbe voluto vendicare l'onore di Madame de Luz. Questo romanzo, dunque, sembrerebbe la prefigurazione de Les malheurs de la vertu di Sade: i due testi possono essere considerati in antitesi al Paméla di Richardson al pari de La religieuse di Diderot, Candide e L'ingénu di Voltaire, in cui le eroine muoiono perseguitate, beffate e oltraggiate senza aver ottenuto giustizia. L'ultimo riferimento a Duclos era insito nel titolo stesso: Mémoires d'une honnête femme écrits par elle-même rimandano alle Mémoires pour servir à l'histoire des mours (1751), in cui l'autore narra in prima persona le vicende della sua vita e, in particolar modo, i suoi incontri galanti con nobildonne dell'epoca.

Diversamente dal romanzo di Duclos, in cui il conte si concede a molteplici amanti prima di rinunciare definitivamente al libertinaggio, l'eroina di Chevrier si dimostra capace sia di deviare i numerosi tentativi di seduzione dei suoi numerosi ammiratori che di rifiutare una proposta di matrimonio una volta rimasta vedova. Julie, infatti, considera il libertinaggio una condotta abominevole in grado di procurarle grande orrore e tremendi sensi di colpa. Nonostante la sua condizione di vedova la renda finalmente padrona del suo destino, Julie sfugge i piaceri mondani scegliendo la via del convento dopo aver constatato il fallimento del libertinaggio in seguito al suicidio del marito, della giovane amante e di due dei suoi spasimanti. I turbamenti amorosi della giovane Julie, infine, permettono di individuare un parallelismo con il romanzo più celebre di Crébillon, Les égarements du cœur et de l'esprit (1736): mentre Meilcour, guidato da Versac, ha avviato la sua iniziazione sentimentale e sessuale, Julie decide di negarsi ai suoi ammiratori fino alla decisione finale di rinchiudersi in convento per evitare qualsiasi relazione amorosa.

Nelle Mémoires d'une honnête femme écrits par elle-même, Chevrier innanzitutto intende mettere in discussione l'educazione religiosa femminile, che egli considera inadeguata perché non prepara le fanciulle alla vita in società e che, 
inoltre, fa rischiare alle ragazze stesse di diventare oggetto dei capricci delle religiose adulatrici. Julie, infatti, percepisce l'inutilità della sua permanenza in convento:

Je vous épargnerai l'ennui du détail des premières années de mon éducation ; vous saurez seulement que des mains de Madame de Verman, mon aïeule, je passai dans un cloître où je fus élevée avec cette fausse austérité qui captive la jeunesse et ne l'instruit point. Jouet perpétuel des caprices des religieuses, je me voyais tout à tour l'objet de leurs tristes complaisances ou de leurs fades plaisanteries. Haïe sans humeur, estimée sans plaisir, le couvent m'ennuyait ; j'en cherchais la raison, et un mouvement secret que je ne pouvais démêler, me disait confusément que le cloître n'était pas fait pour moi (CHEVRIER, 2005: 37).

Allargando la critica sociale, Chevrier mira quindi a mettere in discussione la famiglia tradizionale in termine di poteri coercitivi esercitati dai suoi membri (genitori o fratelli) sulle ragazze ingenue sacrificate in nome degli interessi familiari (come la protagonista Julie e la sua confidente Sophie). Non appena venuta a conoscenza del prossimo matrimonio, Julia manifesta il suo dolore per dover seguire l'obbligo imposto dai genitori:

Je savais bien que l'intérêt réglait la plupart des mariages, mais je me figurais qu'il n'y avait que les filles de Princes qui dussent sacrifier leurs goûts à la politique, et je ne pouvais croire qu'un simple Gentilhomme eût des raisons d'État qui l'obligeassent à devenir le tyran d'une jeune personne dont il devait être l'appui [...]. Devoir austère ! C'est alors qu'accablée sous ton joug j'osais t'accuser de cruauté et d'injustice ! Quoi, donc, me disais-je, esclave du caprice de nos parents, ne pouvons-nous sans crime nous livrer à un penchant fondé sur la raison et la vertu ? Remarques inutiles ! La raison est muette, quand le devoir parle : tout lui cède jusqu'à la vertu même (CHEVRIER, 2005: 38-41).

Dopo aver sposato il conte de Courmont, Julie è costretta a riconoscere i limiti del marito e la sua passione per il cavaliere de Nalbour; al contempo devenascondere il suo disgusto nei confronti dello sposo, al quale è legata dal sacro vincolo coniugale che, però, esclude la passione dal suo cuore:

Tel était Courmont, avec lequel je fus unie sous les auspices marqués par la douleur. Mon mari ne put ce pendant s'apercevoir de mon état, et je fus assez prudente pour lui cacher et ma passion et mes dégoûts. Le Comte était mon époux ; ce titre sacré m'attachait à lui ; si l'amour n'entrait pour rien dans cette union, je n'en étais pas moins sa femme. Le devoir commande aux passions, mais il ne les éteint pas. Je pouvais encore aimer le Chevalier, mais je ne devais être attachée qu'au Comte ; situation équivoque que peu de femmes surmontent! (CHEVRIER, 2005: 42).

Parallelamente alla storia della protagonista Julie, Chevrier descrive il tragico destino che coinvolge un'altra giovane, Sophie, anch'essa educata in convento, impreparata ad affrontare i turbamenti del mondo e costretta a subire le angherie del padre e del fratello. La storia di Sophie è tipograficamente separata da quella di Julie e anticipata da appositi titoli introduttivi ("Histoire de Sophie" e "Suite de l'histoire de Sophie"). Raccontando l'epilogo drammatico della giovane Sophie, Chevrier vuole mostrare la nascita di relazioni incestuose per mettere in discussione la natura 
dei vincoli familiari nella Francia dell'Ancien Régime, la cui degradazione è capace di generare tragici eventi: nella Francia settecentesca infatti soltanto il primogenito ha diritto all'eredità della famiglia mentre i figli cadetti sono prematuramente allontanati dalla famiglia fin dalla tenera età, finendo per dimenticarsi dei parenti diretti, mentre le fanciulle sono costrette a restare in convento per il resto della loro vita (CHAMMAS, 2005: 331-332). Essendo anche lei cresciuta in convento, Sophie non è in grado di riconoscere l'aspetto e l'identità del suo fratello minore che, a sua volta allontanato dalla famiglia per volere del padre autoritario, si è arruolato nell'esercito e, successivamente, otterrà il titolo di marchese d'Ivières. L'autorità della figura paterna è infatti incarnata non solo dal padre di Sophie, ma anche dal fratello maggiore, che alla morte del padre manifesta il proposito di controllare la sorella decidendo la sua sorte e, successivamente, costringendola a prendere forzatamente $\mathrm{i}$ voti. $\mathrm{E}$ interessante, infine, mettere in risalto che la condanna pubblica è riservata esclusivamente a Sophie, costretta ad espiare in convento il crimine dell'incesto, mentre al fratello è permesso di espatriare. J. Chammas sintetizza così la storia di Sophie accusando la sua famiglia di essere la causa dell' avvenuto incesto:

Dans Mémoires d'une honnête femme, Sophie raconte sa propre histoire. Aimée de d'Argis, elle se voit refuser le mariage par son père, qui décide de l'enfermer au couvent contre son gré. Il entend laisser son héritage entier à son fils aîné, après avoir relégué son cadet à un précepteur sans jamais demander à le voir. En route pour le couvent, la voiture est attaquée par d'Argis, qui est aussitôt tué par le père; celui-ci est assassiné à son tour par des brigands. Sophie se retrouve seule à la merci des malfaiteurs, mais elle est sauvé par le Marquis d'Ivières, qui, justement, passait par là. Sophie commente les événements au fur et à mesure qu'ils se produisent, en anticipant les sensations fortes que le lecteur ressentira bientôt. Elle nourrit ainsi sa colère contre le démantèlement de la famille, du fait des retombées effroyables qu'il peut causer : "Je ne me suis sauvée d'un crime que pour frémir sur un autre, bien plus affreux" (CHAmmAs, 2005: 333).

Come Duclos elabora alcune conclusioni generali di carattere filosofico, Chevrier analogamente critica non solo i costumi libertini corrotti, ma anche il perseguimento estremo della virtù in grado di generare tragedie al pari del libertinaggio: Julie si infiamma solamente al pensiero dei suoi amanti, ma quando l'oggetto del suo desiderio diventa accessibile, nega il suo amore divenendo la causa del suicidio di due spasimanti (BOKOBZA-KAHAN, 2005: 23-24). Chevrier ha, dunque, deciso di dar voce alle vicende drammatiche che coinvolgono Julie e, secondariamente, l'amica Sophie per mettere in discussione la costituzione tradizionale della famiglia in grado non solo di imporre ordini autoritari a discapito delle figlie e della moglie (Julie), ma anche di generare tragedie come l'incesto (Sophie). 


\section{BIBLIOGRAFIA}

BOKOBZA KAHAN Michelle (2005), Introduction aux Mémoires d'une honnête femme écrits par elle-même, in : CHEVRIER François-Antoine, Mémoires d'une honnête femme écrits par elle-même, Saint-Étienne, Publications de l'Université de Saint-Étienne, p. 9-24.

ChAMMAS Jacqueline (2005), Confusions familiales et déroutes incestueuses dans quelques romans du milieu du siècle : Caylus, Chevrier, Pernetti, EighteenthCentury Fiction, 17, p. 331-338.

CHEVRIER François-Antoine (2005), Mémoires d'une honnête femme écrits par elle-même, Saint-Étienne, Publications de l'Université de Saint-Étienne.

DE GoncourT Edmond et Jules (1862), La femme au XVIII siècle, Paris, FirminDidot frères.

MATTHEWS-GRIECO Sara (2005), Corps et sexualité dans l'Europe d'Ancien Régime, in : CORBIN Alain; COURTINE Jean-Jacques ; VIGARELLO Georges (éd), Histoire du corps, De la Renaissance aux Lumières, Paris, Seuil, p. 167234.

JATON Anne-Marie (1982), Libertinage féminin, libertinage dangereux, in : PoMEAu René (éd.), Laclos et le libertinage, Paris, P.U.F., p. 151-162.

RUSTIN Jacques (1979), Le vice à la mode : Étude sur le roman français de la première partie du XVIII siècle, Paris, Éditions Ophrys. 